\title{
Love and death: microglia, NLRP3 and the Alzheimer's brain
}

Cell Research (2013) 23:595-596. doi:10.1038/cr.2013.24; published online 12 February 2013

Microglia were previously attributed to be vital brain guardians for neuronal survival and synaptic pruning during development as well as for the brain's fight against environmental pathogens. A new report in Nature by the Heneka, Latz and Golenbock groups, however, sheds new light on these distinct myeloid cells by revealing their deadly nature for mature neurons during neurodegeneration.

Size is frequently equal to significance and beauty, but many good things come in small packages. Thus, one of the smallest populations of brain cells, microglia (named after the Greek micro, meaning "small" because their soma diameter is approximately $12 \mu \mathrm{m}$, and glia, meaning "glue") that only represent $5 \%-10 \%$ of all cells in the central nervous system (CNS) were underappreciated for a long time.

Microglia are truly Janus-faced cells with diverse context-dependent functions and have a close relationship to the hematopoietic system that makes them unique in the CNS [1]. The origin of microglia from the yolk sac (YS), which was already proposed in 1999 [2], could be confirmed by recent fate mapping studies [3, 4]. However, these studies focused on already committed YS myeloid cells and thus considered immature macrophages as microglia precursors, thereby missing possible earlier progenitors lacking macrophage markers such as F4/80 and CD11b. This dilemma was solved in a very recent study in which $\mathrm{CD} 1 \mathrm{~b}^{-}$ c-kit ${ }^{+}$erythromyeloid precursors were identified as the genuine microglia progenitors at a stem cell level [5].

Alzheimer's disease (AD) is a severe and prevalent neurodegenerative disorder that compels an intensive research effort directed at understanding its pathophysiology and treatment. At present, no established treatment ameliorates the natural course of AD. The essential neuropathological characteristic of $\mathrm{AD}$ comprises the aggregation and accumulation of intracellular and extracellular components. Specifically, $\mathrm{AD}$ brains contain senile plaques composed of extracellular deposits of amyloid peptides (collectively termed amyloid- $\beta, A \beta$ ) derived from amyloid precursor protein (APP). Additionally, neurons in affected regions contain intracellular aggregates (designated neurofibrillary tangles) composed of hyperphosphorylated forms of the microtubule-associated protein tau. During the development of $\mathrm{AD}$, these two processes interact in poisonous succession. The neuropathological findings demonstrate that the CNS tissue reacts vigorously to a multifaceted assault comprised of $A \beta$ peptide oligomers, amyloid fibrils and plaques, tau fibrils and neurofibrillary tangles, synaptic dysfunction, neuronal cell impairment and cell death [6].

What is the precise function of microglia during $\mathrm{AD}$ and how do they mediate pathology? These important questions were addressed by a seminal study published recently in Nature [7] and microglia were presented as potential therapeutic targets for selective drug applications in $\mathrm{AD}$ patients.
The authors focused on the role of the inflammasome in microglia during $\mathrm{AD}$ progression (Figure 1). The inflammasome is an intracellular multimolecular complex required for the activation of inflammatory caspases, which orchestrates the cleavage and secretion of interleukin (IL)-1 $\beta$, IL-18 and IL-33, thereby generating a potent inflammatory response [8]. The nucleotide binding and oligomerization domainlike receptor family pyrin domain containing 3 (NLRP3) inflammasome, composed of NLRP3, the adaptor molecule apoptosis-associated speck-like protein containing a caspase recruitment domain (Asc), and the cysteine protease caspase-1, has been implicated in several chronic inflammatory diseases as it can sense inflammatory crystals and aggregated proteins, including $A \beta$ [9]. The authors elegantly provided evidence for the increase in cleaved caspase-1 in AD patients, indicating disease-associated inflammasome activation. Using APP/PS1 transgenic mice that express a human/ mouse chimeric APP and human presenillin-1(PS1), each carrying familial AD-linked mutations, the authors elucidated the role of the inflammasome in $\mathrm{AD}$ pathogenesis in vivo. Brain endogenous NLRP3 inflammasome activation was restricted to plaqueassociated microglia and absent in all other CNS cells, as clearly shown by microglia-specific activation of Asc. NLRP3- or caspase-1-deficient mice showed significantly reduced amyloid burden and clearly improved cognitive impairment as assessed by a bat- 


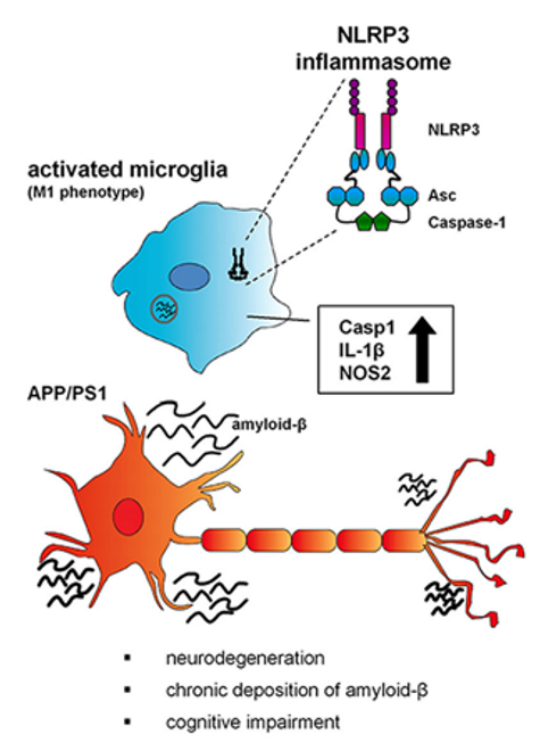

Figure 1 Proposed mechanism of inflammasome-mediated neurotoxicity in AD. Microglia are equipped with the intracellular multimolecular NLRP3 complex, of which activation by $A \beta$ in APP/PS1 AD mice induces an activated M1-related phenotype that enhances neuronal loss, $A \beta$ deposition and cognitive decline. In contrast, microglia-specific disruption of functional NLRP3 inflammasome strongly reduces extracellular $A \beta$ load, preserves the neurons and protects against cognitive impairment. Arg1, arginase-1; Casp1, caspase-1; FIZZ1, found in inflammatory zone 1; NOS2, nitric oxide synthase 2 .

tery of spatial memory and behavioral tests. Ameliorated cognitive dysfunction and normalized behavior was attributed to the restoration of long term potentiation, which indicates memory formation, and preserved neuronal spine morphology. One can therefore assume that $A \beta$ elicits a cleavage of caspase-1 and production of cytotoxic IL-1 $\beta$ in a NLRP3-dependent manner that acts in an autocrine way to amplify neurotoxicity through the induction of other proinflammatory mediators in vivo. NLRP3 activation by $\mathrm{A} \beta$ was previously linked to phagocytosis, as the inhibition of phagocytosis led to a decrease in NLRP3-mediated release of IL-1 $\beta$ in vitro [9]. In fact, triple transgenic APP/PS1/Nlrp3 $3^{-1-}$-mice showed altered phagocytotic activity in microglia where $A \beta$ uptake was enhanced. These data provide compelling evidence that microglia-specific activation of the inflammasome is pivotal for the pathogenesis of $\mathrm{AD}$. Whether naturally occurring mutations of the inflammasome complex, such as pyrinlike protein mutations, also modify neurodegenerative disorders besides inducing severe autoinflammatory syndromes [10] remains to be investigated. In addition, the mechanisms for sensing $A \beta$ by the intracellular ligand finally activating the NLRP3 inflammasome in microglia is still an open question. Taken together, this seminal study by Heneka and colleagues revealed the microglia-specific inflammasome as a promising cell type-specific molecular target in the CNS for therapeutic intervention for AD.

\section{Tobias Goldmann ${ }^{1}$, Tuan Leng Tay ${ }^{1}$, Marco Prinz ${ }^{1,2}$}

${ }^{1}$ Institute of Neuropathology, University of Freiburg, Germany; ${ }^{2}$ BIOSS Center for Biological Signalling, University of Freiburg, Germany Correspondence: Marco Prinz

Tel: 49-761-270-5105; Fax: 49-761-270-5050

E-mail: marco.prinz@uniklinik-freiburg.de

\section{References}

1 Prinz M, MildnerA. Glia 2011; 59:177-187.

2 Alliot F, Godin I, Pessac B. Brain Res Dev Brain Res 1999; 117:145-152.

3 Ginhoux F, Greter M, Leboeuf M, et al. Science 2010; 330:841-845.

4 Schulz C, Gomez Perdiguero E, Chorro L, et al. Science 2012; 336:86-90.

5 Kierdorf K, Erny D, Goldmann T, et al. Nat Neurosci 2013; 16:273-280.

6 Prinz M, Priller J, Sisodia SS, et al. Nat Neurosci 2011; 14:1227-1235.

7 Heneka MT, Kummer MP, Stutz A, et al. Nature 2013; 493:674-678.

8 Franchi L, Eigenbrod T, Munoz-Planillo R, et al. Nat Immunol 2009; 10:241-247.

9 Halle A, Hornung V, Petzold GC, et al. Nat Immunol 2008; 9:857-865.

10 Hoffman HM, Mueller JL, Broide DH, et al. Nat Genet 2001; 29:301-305. 